\title{
Lipocortin 1: glucocorticoids caught in the act?
}

\author{
Susan F Smith
}

\begin{abstract}
Glucocorticoids (glucocorticosteroids, corticosteroids) have an important place in the treatment of many inflammatory conditions including those of the respiratory tract. Their mechanisms of action include both the suppression of proinflammatory mediators and the upregulation of at least one anti-inflammatory protein, lipocortin 1 (also known as annexin 1). Lipocortin 1 has been convincingly demonstrated to mediate the anti-inflammatory effects of glucocorticoids in a variety of in vivo and in vitro models of inflammation. The actions of lipocortin 1 in the lung have not been fully elucidated. If, as initial studies suggest, its effects in the respiratory tract are shown to be anti-inflammatory, it is possible that administration of lipocortin 1 peptides, or other drugs based on the active site of lipocortin 1 , might prove to be useful agents for the control of respiratory tract inflammation.

(Thorax 1996;51:1057-1059)
\end{abstract}

Keywords: lipocortin 1, glucocorticoid, respiratory tract.

Distribution and glucocorticoid inducibility of lipocortin 1

The glucocorticoids are drugs of enormous therapeutic importance yet, like many other agents in wide clinical use, their precise mechanism(s) of action remain incompletely understood. Because endogenously produced glucocorticoids have important metabolic functions, when administered in "pharmacological" doses for their anti-inflammatory actions this class of drug has many side effects, ranging from the inconvenient to the life-threatening. For this reason there is considerable interest in finding safer but effective new antiinflammatory agents which can be used as alternatives or in addition to current therapeutic regimens, so reducing the requirement for glucocorticoids.

It is clear that the anti-inflammatory action of glucocorticoids is multifaceted. Many different components of the inflammatory response are suppressed by the direct or indirect modulation of transcription of specific target genes. Although most of these actions result in a reduction in level or activity of pro-inflammatory mediators, a few anti-inflammatory proteins are thought to be upregulated by gluco- corticoids; one of which - lipocortin 1 (also known as annexin 1) - is the subject of this review. Lipocortin 1 is a member of the family of annexins which are calcium and phospholipid binding proteins with a shared core structure but considerable diversity of the $\mathrm{N}$-terminal region. Each member of the family, which currently numbers at least 13 , has a different cellular distribution and probably a unique biological function. ${ }^{1}$

Lipocortin 1 is widely distributed, but is particularly abundant in the lung where it is found in epithelial cells, tracheal gland cells, alveolar macrophages and neutrophils and, to a lesser extent, in vascular smooth muscle (fig 1). ${ }^{2}$ In vitro studies have shown that it is glucocorticoid inducible in both alveolar macrophages and type II epithelial cells (fig 1). Studies in humans have shown that levels of lipocortin 1 increase in the respiratory tract secretions of healthy volunteers ${ }^{3}$ and lung lavage cells of patients ${ }^{4}$ following oral administration of therapeutic doses of glucocorticoid, suggesting that this protein is, indeed, steroid inducible in the human lung. Interestingly, smokers have higher levels of extracellular lipocortin 1 in their lung lavage fluid than non-smokers. ${ }^{5}$ This smoke-related increase in extracellular lipocortin 1 (which could represent either active secretion or release due to cell damage) is likely to replenish protein inactivated by the high levels of neutrophil elastase found in smokers and shown previously to cleave lipocortin $1 .^{6}$

\section{Scientific basis}

BIOLOGICAL ACTIVITY OF LIPOCORTIN 1

The first experiments to demonstrate the existence of the protein were performed independently in three different laboratories each of which showed, in a different in vitro model system, that an anti-inflammatory protein appeared to mediate the suppression by glucocorticoids of phospholipase $\mathrm{A}_{2}\left(\mathrm{PLA}_{2}\right)$ activity and subsequent eicosanoid synthesis. These studies stimulated a wave of excitement that this protein, which was named lipocortin, might be "the" mechanism of action of glucocorticoids. This was swiftly followed by a period of disillusionment when a number of groups tried without success to demonstrate anti-inflammatory activity of the recombinant and native proteins, both in vivo and in vitro. A major turning point in the rehabilitation of lipocortin 1 into polite, scientific society was 
the discovery that the recombinant protein required refolding into the correct threedimensional conformation in order to be biologically active.$^{7}$ Furthermore, it was found that purified lipocortin 1 is readily denatured at airliquid interfaces. As the preparation of solutions for injection or mixtures for incubation nearly always includes at least one bout of vortex mixing, most researchers had therefore been attempting to perform the scientific equi-valent of hatching chicks from hard boiled eggs - not surprising then that the number of successful experiments was only slightly greater than the number of successful hatchings!

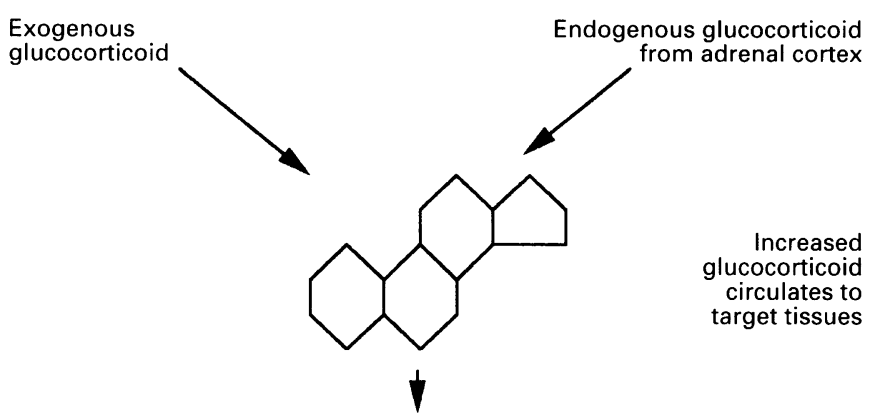

Target cells include alveolar macrophages and epithelial cells in the lung and neutrophils in blood
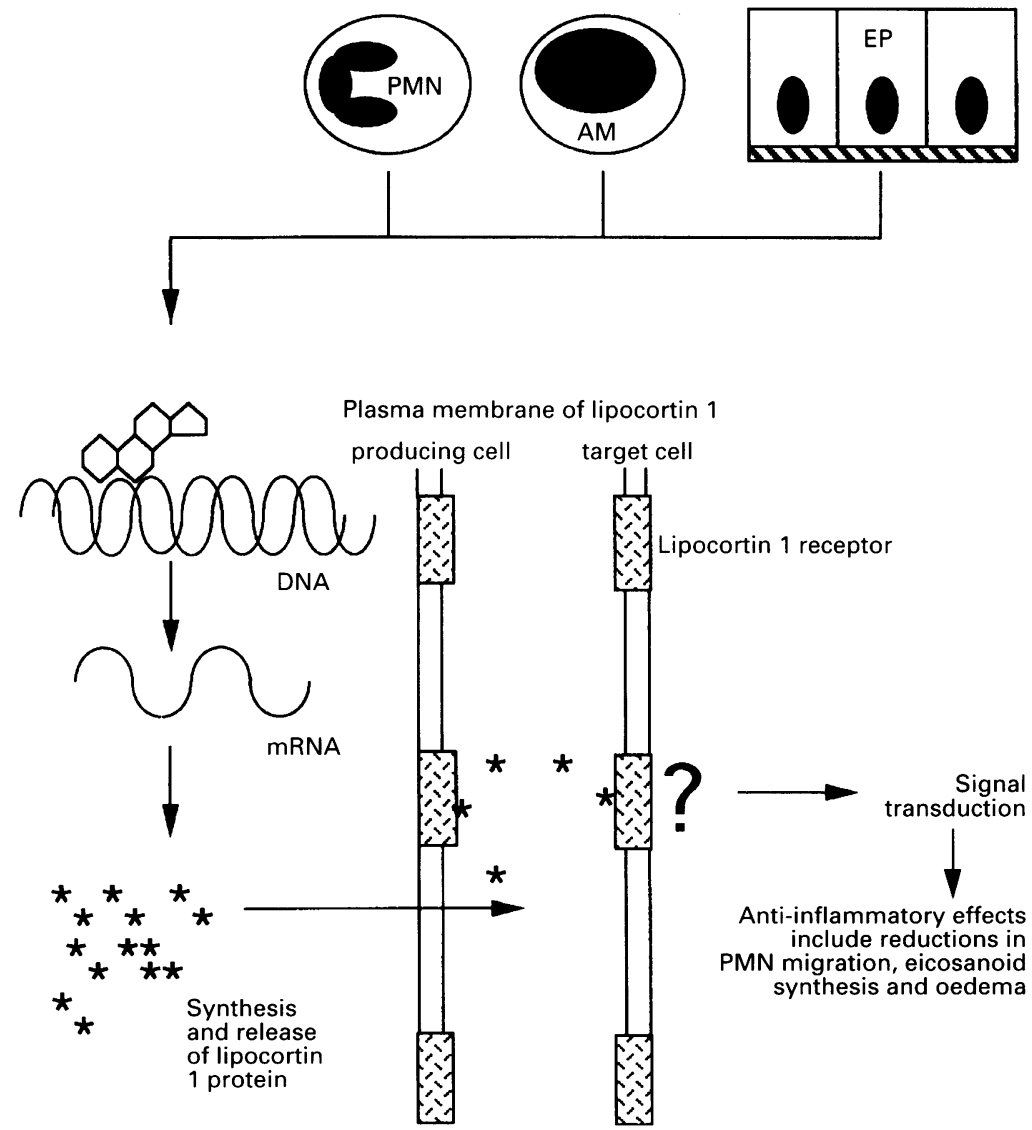

Figure 1 Circulating endogenous or synthetic glucocorticoid stimulates target cells to increase synthesis and/or release of lipocortin 1 protein. Released lipocortin 1 (depicted as *) attaches to cell surface binding sites (hatched rectangles) on the target cells. Its mode of action may be autocrine or paracrine and thus a lipocortin 1 producing cell may also be a target for the protein following its release. Details of the steps between receptor binding and the cellular anti-inflammatory response remain to be established and are summarised by the question mark (?). PMN=polymorphonuclear neutrophil; $A M=$ alveolar macrophage; $E P=$ type II epithelial cell.
These revelations stimulated the development of powerful new tools with which to examine the actions of lipocortin 1. Firstly, synthetic peptides derived from the primary sequence of the protein and sharing its activity, but possessing enhanced stability and, secondly, antibodies which neutralise lipocortin 1 activity in vivo and in vitro. The use of these tools has allowed clear demonstration of the biological activity of both exogenously administered and endogenously produced lipocortin 1. For example, there is now convincing evidence from various experimental models that exogenous lipocortin 1 and lipocortin 1 peptides mimic, and neutralising antilipocortin 1 antisera abrogate, the suppression by glucocorticoids of several aspects of the inflammatory response including neutrophil migration and the development of oedema and fever. ${ }^{89}$

Although the lung is rich in lipocortin 1 and has been used in many studies as a source for the isolation of the protein, to date few studies have investigated its biological activity in the respiratory tract. However, neutralising antibodies to lipocortin 1 have been shown to reverse at least one action of glucocorticoid in the lung - namely, the downregulation by dexamethasone of inducible nitric oxide synthase (iNOS) expression in the lung of lipopolysaccharide-treated rats. ${ }^{10}$

MECHANISM OF ACTION OF LIPOCORTIN 1

Having established that lipocortin 1 possesses anti-inflammatory activity, the next question is obviously "How does it work?" Using a fluorescence activated cell sorter (FACS) and fluorescently tagged antibodies for detecting the protein, it has been shown that lipocortin 1 occupies a specific cell surface binding site on the plasma membrane of the target cell, ${ }^{11}$ thus indicating that extracellular release is essential for the activity of this protein (fig 1). How this interaction between lipocortin 1 and its binding site triggers a reduction in inflammation is not entirely clear. It is known that in the A549 cell line lipocortin 1 reduces eicosanoid production, not by altering expression of cyclo-oxygenase-2 (COX-2) but by reducing $\mathrm{PLA}_{2}$ activity, perhaps as a result of modulation of signal transduction processes. ${ }^{12}$ Whether or not this is the case in all cells remains to be determined (fig 1).

As in other species, the mechanism of action of lipocortin 1 in the human lung remains to be fully elucidated. Interestingly, an inverse relationship has been shown between the levels of lipocortin 1 and prostaglandin $\mathrm{D}_{2}$ (which can cause bronchoconstriction) in lung lavage fluid from patients with allergic asthma. ${ }^{5}$ At the same time this was associated with an increase in the metabolite of prostacyclin (which can protect against bronchoconstriction), giving rise to the intriguing but unproven possibility that lipocortin 1 modifies the balance between the different prostanoids produced. 


\section{Therapeutic potential}

It is now clear that lipocortin 1 has anti-inflammatory activity. How this can be harnessed therapeutically is less obvious. Potential routes of administration are limited for a protein and, given its known instability ex vivo, use of intact lipocortin 1, recombinant or native, seems unlikely. Synthetic peptides based on specific regions of the protein sequence have greater stability but less biological activity on a molar basis. As soon as more is known about the mechanism of action of lipocortin 1 and about the chemistry of the interaction with its binding site, it may prove possible to design new, nonprotein based drugs with structures which confer the capacity to stimulate the binding site in a similar way to lipocortin 1 itself.

\section{Conclusion}

A lipocortin 1-based therapeutic agent might prove to be a useful adjunct to glucocorticoid therapy, perhaps allowing the reduction of the doses of glucocorticoid administered or, if given with an appropriate cocktail of anti-inflammatory agents, it might even replace the glucocorticoid in the treatment of certain lung diseases.

The author would like to thank Professor R J Flower and Dr $\mathrm{T} D$ Tetley for their helpful comments on the manuscript.
1 Moss SE. The annexins. In: Moss SE, ed. The annexins. London: Portland Press, 1992:1-10.

2 Fava RA, McKanna J, Cohen SI. Lipocortin 1 is abundant in a restricted number of differentiated cell types in adult organs. F Cell Physiol 1989;141:284-93.

3 Smith SF, Tetley TD, Datta AK, Smith T, Guz A, Flower RJ. Lipocortin-1 distribution in bronchoalveolar lavage from healthy human lung: effect of prednisolone. $\mathcal{J} A p p l$ Physiol 1995;79:121-8.

4 De Caterina R, Sicari R, Giannessi D, Paggiaro PL, Paoletti $\mathrm{P}$, Lazzerinin, et al. Macrophage-specific eicosanoid synthesis inhibition and lipocortin-1 induction by glucothesis inhibition and lipocortin-1 induction

5 van Hal PThW, Overbeek SE, Hoogsteden HC, Zijlstra FJ, Murphy K, Oosterhoff Y, et al. Eicosanoids and lipocortin1 in BAL fluid in asthma: effects of smoking and inhaled glucocorticoids $\mathcal{F}$ Appl Physiol 1996 (in press).

6 Smith SF, Tetley TD, Guz A, Flower RJ. Detection of lipocortin 1 in human lung lavage fluid; lipocortin degradation as a possible proteolytic mechanism in the control of inflammatory mediators and inflammation. Environ Health Perspect 1990;85:135-44.

7 Browning JL, Ward MP, Wallner BP, Pepinsky RB. Studies on the structural properties of lipocortin-1 and the regulation of its synthesis by steroids. In: Melli M, Parente $\mathrm{L}$, eds. Cytokines and lipocortins in inflammation and differentiation. New York: Wiley-Liss, 1990: 27-45.

8 Flower RJ, Rothwell NJ. Lipocortin 1: cellular mechanisms and clinical relevance. Trends Pharmacol Sci 1994;15:71-6.

9 Perretti M. Lipocortin-derived peptides. Biochem Pharmacol 1994;47:931-8.

10 Wu C-C, Croxtall JD, Perretti M, Bryant CE, Thiermermann C, Flower RJ, et al. Lipocortin 1 mediates the inhibition of dexamethasone of the induction by endotoxin of nitric oxide synthase in the rat. Proc Natl Acad Sci USA 1995;92:3473-7.

11 Goulding NJ, Luying P, Guyre PM. Characteristics of lipocortin 1 binding to the surface of human peripheral blood leukocytes. Biochem Soc Trans 1990;18:1237-8.

12 Croxtall JD, Choudhury Q, Tokumoto H, Flower RJ Lipocortin-1 and the control of arachidonic acid release in cell signalling. Glucocorticoids inhibit $G$ protein-dependent activation of $\mathrm{CPLA}_{2}$ activity. Biochem Pharmacol 1995;50:465-74. 